\title{
Erratum
}

\section{Preinjury employment status as a risk factor for symptomatology and disability in mild traumatic brain injury: A TRACK-TBI analysis}

[NeuroRehabilitation 43(2) (2018), pp. 169-182; DOI: 10.3233/NRE-172375]

https://content.iospress.com/articles/neurorehabilitation/nre172375

When this article was originally published, due to an author error, one of the author affiliations was missing. The correct list of authors and affiliations is given here:

John K. Yue ${ }^{\mathrm{a}, \mathrm{b}}$, Jonathan W. Rick ${ }^{\mathrm{a}, \mathrm{b}}$, Molly Rose Morrissey ${ }^{\mathrm{a}, \mathrm{b}}$, Sabrina R. Taylor ${ }^{\mathrm{a}, \mathrm{b}}$, Hansen Deng ${ }^{\mathrm{a}, \mathrm{b}}$, Catherine G. Suen ${ }^{\mathrm{a}, \mathrm{b}}$, Mary J. Vassar ${ }^{\mathrm{a}, \mathrm{b}}$, Maryse C. Cnossen ${ }^{\mathrm{c}}$, Hester F. Lingsma $^{\mathrm{c}}$, Esther L. Yuh ${ }^{\mathrm{b}, \mathrm{d}}$, Pratik Mukherjee ${ }^{\mathrm{b}, \mathrm{d}}$, Raquel C. Gardnere, ${ }^{\mathrm{e}}$, Alex B. Valadka ${ }^{\mathrm{g}}$, David O. Okonkw ${ }^{\mathrm{h}}$, Tene A. Cage ${ }^{\mathrm{a}, \mathrm{b}}$, Geoffrey T. Manley ${ }^{\mathrm{a}, \mathrm{b}}$, and the TRACK-TBI Investigators

${ }^{a}$ Department of Neurological Surgery, University of California San Francisco, San Francisco, CA, USA

${ }^{\mathrm{b}}$ Brain and Spinal Injury Center, Zuckerberg San Francisco General Hospital, San Francisco, CA, USA

${ }^{\mathrm{c}}$ Department of Public Health, Erasmus Medical Center, Rotterdam, The Netherlands

${ }^{\mathrm{d}}$ Department of Radiology, University of California San Francisco, San Francisco, CA, USA

${ }^{\mathrm{e}}$ Department of Neurology, University of California San Francisco, San Francisco, CA, USA

${ }^{\mathrm{f}}$ Department of Neurology, Veterans Affairs Medical Center, San Francisco, CA, USA

${ }^{\mathrm{g}}$ Department of Neurological Surgery, Virginia Commonwealth University, Richmond, VA, USA

${ }^{\mathrm{h}}$ Department of Neurological Surgery, University of Pittsburgh, Pittsburgh, PA, USA 